\title{
Perspective
}

PERSPECTIVE Actualité en histoire de l'art

4 | 2007

Genre et histoire de l'art

Pratique, histoire et théorie de l'art féministe aux États-Unis en 2007 : les expositions Wack ! et Global Feminisms

\section{Elvan Zabunyan}

\section{(2) OpenEdition \\ Journals}

Édition électronique

URL : http://journals.openedition.org/perspective/3584

DOI : 10.4000/perspective.3584

ISSN : 2269-7721

Éditeur

Institut national d'histoire de l'art

Édition imprimée

Date de publication : 31 décembre 2007

Pagination : 731-737

ISSN : 1777-7852

Référence électronique

Elvan Zabunyan, « Pratique, histoire et théorie de l'art féministe aux États-Unis en 2007 : les expositions Wack! et Global Feminisms », Perspective [En ligne], 4 | 2007, mis en ligne le 31 mars 2018, consulté le 01 octobre 2020. URL : http://journals.openedition.org/perspective/3584 ; DOI : https:// doi.org/10.4000/perspective.3584 


\title{
Pratique, histoire et théorie de l'art féministe aux États-Unis en 2007 : les expositions Wack! et Global Feminisms
}

\author{
Elvan Zabunyan
}

1 Les liens tissés entre l'art et le féminisme dès la fin des années 1960 sont (re)découverts aujourd'hui à travers les expositions internationales étudiées ici mais également, grâce à d'ambitieuses anthologies qui, réunissant les essais majeurs produits par les artistes, critiques et historien-e-s de l'art féministes, témoignent de la vivacité d'une création et d'une réflexion qui gardent toujours, quarante ans après, une véritable dynamique ${ }^{1}$.

2 La relation entre le temps présent et le temps historique est aussi à souligner au regard de l'engagement des générations nées précisément dans les années 1950 ou 1960 qui, en interrogeant les œuvres, les actions et les documents qui leur ont été légués par les féministes des années 1960-1970, les intègrent dans une nouvelle historiographie, les utilisent comme des outils méthodologiques et surtout tentent de combler, grâce à eux, la vacuité de la pensée politique de notre temps. Il nous semble en effet que l'importance croissante de ces questions féministes, ainsi que celles, complémentaires, des gender et queer studies, pour la sphère des sciences humaines, politiques et sociales, découle de la volonté de repenser la valeur des différences de sexe, race et classe et, en écoutant avec attention cette histoire intellectuelle et culturelle, de continuer à la faire vivre en la transmettant ${ }^{2}$.

3 Cet héritage est précisément celui qui fut discuté lors du colloque The Feminist Future: Theory and Practice in the Visual Arts qui s'est tenu au Museum of Modern Art (MoMA) de New York en janvier 2007. Les deux journées d'interventions et de discussions étaient regroupées selon les problématiques suivantes : «Activism/Race/Geopolitics », «Body/ Sexuality/Identity ", "Writing the History of Feminism ", "Institutionalization of Feminism ». Dans sa conférence inaugurale, Lucy Lippard affirmait que la force du 
féminisme vient de la multiplicité de ses définitions, maintenant vif l'esprit d'une réelle culture féministe ${ }^{3}$.

4 En faisant intervenir des personnalités impliquées dans la construction du rapport de l'art au féminisme des dernières décennies, le colloque se voulait un espace de réflexion analysant, quatre décennies après ses premiers pas, les ouvertures (mais aussi les limites) du féminisme et de son influence incontestable sur la création artistique contemporaine depuis cette époque. L'une des limites que l'on retiendra à l'écoute de ces interventions et à la lecture des essais dans les catalogues d'exposition de WACK! et de Global Feminisms ${ }^{4}$ est le retard avec lequel les féministes blanches ont commencé à intégrer dans leurs analyses les problèmes de discrimination raciale subies par leurs consœurs « de couleur $»^{5}$. Ainsi, si la question du sexisme était posée par les unes, elle était indissociable du racisme pour les autres ${ }^{6}$.

5 Très tôt engagée dans les problématiques alors appelées multiculturelles faisant logiquement suite au mouvement des droits civiques américains, Lucy Lippard (qui milite, en 1970, avec l'artiste africaine américaine Faith Ringgold) reconnaît en effet les impasses de la "seconde vague féministe " (la "première vague » renvoyant aux suffragettes du début $d u \mathrm{xx}^{\mathrm{e}}$ siècle) qui se définit avant tout comme appartenant à la culture blanche des classes moyenne et bourgeoise américaines et relègue à l'arrièreplan la réalité des femmes "de couleur» issues des classes populaires. Aussi les expositions WACK! Art and The Feminist Revolution et Global Feminisms tentent-elles de présenter un visage différent de ce féminisme historique notamment grâce à une ouverture au-delà des frontières américaines et européennes. Les commissaires affirment dans leurs notes d'intention que les formes de création et de revendications non seulement ne peuvent plus se dissocier des différences culturelles et raciales mais doivent, au contraire, les intégrer dans les paradigmes théoriques et critiques mis en œuvre dans la pensée féministe contemporaine.

\section{WACK! Art and the Feminist Revolution, par-delà I'histoire d'un mouvement}

6 Parmi les expositions que cite comme références la commissaire de WACK!, Connie Butler, Bad Girls de Marcia Tucker (New Museum of Contemporary Art, New York, 1994), Inside the Visible: An Elliptical Trace of 20th Century art: In, Of, and From the Feminine de Catherine de Zegher (Institute of Contemporary Art, Boston, 1996) ou encore Sexual Politics d'Amelia Jones (Hammer Museum, Los Angeles 1996) sont celles qu'elle considère comme particulièrement emblématiques des problématiques historiques féministes développées dans les années 1990. Ces dernières s'inscrivent dans le contexte de virulentes revendications contre l'institution muséale, avec la création en $1992 \mathrm{du}$ Women Art Coalition (WAC) qui proteste contre l'absence presque totale d'artistes femmes dans l'exposition inaugurale du Guggenheim dans son bâtiment de Soho la même année. Cet épisode, et les expositions précédemment citées qui l'ont suivi, relance une réflexion qui s'était en quelque sorte mise en veille dans le contexte conservateur des années Reagan. Selon C.Butler, malgré leur importance, ces manifestations n'obtiennent pas, à cette époque, la réception escomptée. C'est à ce moment que l'idée de réaliser une exposition majeure sur l'art et le féminisme germe dans son esprit, et elle consacre alors huit années à ses recherches, décidée à proposer un projet où les artistes américaines ne seront pas majoritaires. À cet effet, dans son 
essai «Chronology through Cartography: Mapping 1970s Feminist Art Globally », qui ouvre la partie théorique du catalogue de WACK! (p.322-335), Marsha Meskimmon tente de considérer la chronologie des pratiques artistiques féministes non plus du seul point de vue temporel, linéaire et eurocentrique mais aussi spatial, géographique et global. L'exposition s'appuie sur la diversité des œuvres (plastiques, photographiques, filmiques, écrites, corporelles, sonores, conceptuelles) réalisées par les artistes, toutes des femmes, entre 1965 et 1980 .

7 Dès son annonce médiatique officielle, la manifestation, outre la curiosité qu'elle suscite, déclenche d'inévitables controverses. L'une est liée à son titre considéré comme ayant une sonorité trop caricaturale. Dans son introduction, Connie Butler explique le choix de celui-ci par une volonté de le mettre en parallèle avec les acronymes des collectifs activistes féministes des années 1970 - WAR (Women Artists in Revolution), WAC (Women's Action Coalition), WCA (Women's Caucus for Art). «WACK!» aurait aussi selon elle une connotation violente, renforçant le potentiel révolutionnaire du féminisme dans sa lutte contre le patriarcat. L'explication ne convainc qu'à moitié car si le terme de "révolution» dans le sous-titre s'apparente certes aux luttes politiques du mouvement des femmes, il s'adresse également à leurs productions qui, par leur caractère innovant et provocant, transforment radicalement les paramètres de lecture de l'art contemporain - l'art des femmes de cette époque est celui qui pose certainement pour la première fois dans l'histoire de l'art une représentation aussi frontale du corps, du sexe et de la sexualité. Or, paradoxalement, rien dans l'exposition, ni cartel, ni chronologie, ni texte explicatif, ni documents visuels (à l'exception du carton d'invitation et de l'affiche de l'exposition) ne vient éclairer le ton exclamatif du titre.

8 Ainsi, dans l'immense espace de Geffen Contemporary (près de 8000 mètres carrés consacrés aux œuvres), ancien hangar réhabilité devenu l'un des lieux d'exposition du MOCA, les visiteurs n'obtiennent aucune information historique qui leur permettrait de comprendre précisément le contexte politique, culturel et social au sein duquel les pièces exposées ont été produites. L'absence de documents d'archives et de chronologie est d'autant plus gênante que beaucoup d'œuvres produites par les artistes femmes l'étaient dans le cadre de collectifs et de communautés artistiques. Les travaux de Judy Chicago ou Miriam Shapiro ne peuvent être dissociés ni de leur premier programme pédagogique - le Feminist Art Program - qui ouvre en 1971 au California Institute of the Arts, ni de l'expérience unique de la Womanhouse, où pendant l'hiver 1972 de nombreuses artistes produisent des installations dans les différentes pièces du bâtiment. C'est là que sont réalisées les performances parmi les plus célèbres de la période féministe telle Waiting de Faith Wilding, que l'artiste réinterprète d'ailleurs pour WACK! lors d'un événement unique le 11 mars 2007 intitulé Wait-with. Pas d'évocation précise non plus de l'ouverture, à Los Angeles, à l'automne 1973, du Women's Building, gigantesque projet entièrement consacré à l'activité et à la recherche des femmes et qui marque un nouveau tournant dans la relation entre pratiques artistiques, ateliers, enseignements, expositions et discussions féministes.

9 Pour WACK!, cent vingt artistes ont été sélectionnées, plusieurs centaines de travaux sont présentés, autant d'heures de vidéos et de films sont proposées et la visite nécessite plusieurs journées pour être effective. Alors que la période choisie par C. Butler recouvre précisément les années phares d'une histoire de l'art féministe, le choix est fait de montrer les œuvres rassemblées sous différentes thématiques: Gender 
performance (Adrian Piper, Cindy Sherman), Abstraction (Senga Nengudi; Miriam Shapiro), Gendered Space (Eva Hesse, Louise Bourgeois), Body As Medium (Carolee Schneemann, Valie Export), Knowledge as Power (Judy Chicago), Collective Impulse (Kirsten Dufour, Where We At Black Women Artists), Social Sculpture (Lygia Clark, Suzanne Lacy), Silence and Noise (Theresa Hak Kyung Cha, Ketty La Rocca), Speaking in Public (Faith Ringgold, Cosi Fanni Tutti), Making Art History (Mary Beth Edelson, Léa Lublin), Taped and Measured (Eleanor Antin, Martha Rosler), Autophotography (Hannah Wilke, Barbara Hammer), Female Sensibility (Lynn Hershmann, Yvonne Rainer), Pattern and Assemblage (Betye Saar, Howardena Pindell), Body Trauma (Nancy Grossman, Ana Mendieta), Goddess (Niki de Saint-Phalle, Lorraine O'Grady, Orlan), Family Stories (Linda Montano, Lesbian Art Project), et enfin Labor (Mary Kelly, Mierle Laderman Ukeles) ${ }^{7}$. Une analyse plus précise de ces thématiques révélerait, pour certaines, leur nature stéréotypée et superficielle s'opposant à la justesse et à la radicalité des œuvres, et montrerait à quel point elles peuvent parfois contredire la réalité même des pratiques de l'époque. Celles-ci, tentant justement de se distancer de toute catégorisation, cherchaient à exprimer sans contrainte les problématiques inhérentes à la création, remettant en cause les clichés associés au statut de la femme jusqu'à les faire céder. L'aspect restrictif de la thématique se retrouve aussi dans un accrochage trop muséal qui empêche les courants de circuler librement. Cloisonnées par les thèmes, les œuvres le sont aussi par des cimaises qui rendent leur lecture parfois difficile. Alors que l'étonnante richesse de l'art féministe est largement visible, les interactions entre les différentes actrices du mouvement et les correspondances explicites entre les cultures américaines et européennes sont absentes.

10 La relation au travail, leitmotiv de cette époque et devenu à ce titre "thématique ", recouvre aussi bien le labeur domestique que la maternité. Cette dernière est par exemple interrogée par la fameuse série de Mary Kelly, Post Partum Document, 1973-1975 qui documente, jour après jour, l'évolution de son nouveau-né et qui est à la fois un travail conceptuel sur le texte et un méticuleux rapport d'une mère qui répertorie la répétition de ses tâches quotidiennes selon un modèle de production de masse. Ce travail répond à une exposition, Women and Work: A Document on the Division of Labour in Industry, 1973-1975 (1973-1975), que l'artiste a réalisée avec Margaret Harrison et Kay Hunt aux mêmes dates que Post Partum. Présentée à Londres en 1975, elle est réinstallée à l'occasion de WACK!.

11 La documentation sérielle est aussi celle qui nous permet de découvrir pour la première fois dans son intégralité la performance de Mierle Laderman Ukeles, Washing/Tracks/ Maintenance: Inside, July 22, 1973 (1973) et Washing/Tracks/Maintenance: Outside, July 22, 1973 (1973) dont le projet est de littéralement nettoyer l'intérieur et l'extérieur de tous les musées où est montrée l'exposition conceptuelle itinérante intitulée $c, 7,500$ dont Lucy Lippard est la commissaire. Le 22 juillet 1973, comme on le voit sur les photographies présentées, M. Laderman Ukeles fait le ménage au Wadsworth Atheneum à Hartford dans le Connecticut. Son introduction en tant qu'artiste dans le musée se fait donc par une critique de l'institution où femmes de ménage, gardiens et ouvriers occupent une place subalterne. Dès lors, cette performance articule autant une critique de classe qu'une critique de la condition des femmes dans leur rôle domestique.

12 L'utilisation de la série photographique est récurrente chez beaucoup d'artistes féministes : ainsi, en 1972, Eleanor Antin endure pendant trente-six jours un régime 
amaigrissant et perd cinq kilos. Elle réalise chaque jour un autoportrait en pied (profil gauche, profil droit, face, dos) montrant la lente transformation de son corps nu. Les soixante-douze photographies sont présentées sous la forme d'une grille qui permet de comparer les différentes étapes de son amaigrissement. Ironiquement intitulée Carving: A Traditional Sculpture, l'œuvre renvoie simultanément à l'espace social et à l'académie, à la condition féminine et à la statuaire, qui doivent répondre dans les deux cas à des canons de représentations normatifs.

13 Si la grande qualité de WACK! est de montrer des œuvres qui, pour la plupart, n'ont presque jamais été exposées depuis leur création, néanmoins, malgré le nombre louable d'artistes choisies, la question de la sélection se pose, notamment au regard de la volonté première du projet qui était de révéler des artistes non-américaines et issues des courants minoritaires. Si les artistes européennes sont majoritairement présentes, trop peu d'artistes "de couleur» le sont et la manifestation reste également très orientée vers un féminisme hétérosexuel alors que la culture homosexuelle est l'une de celles qui a permis l'ouverture vers les problématiques de gender telles qu'elles sont étudiées aujourd'hui'. Dans le même temps, la critique concernant ces deux points renvoie assez clairement à la réalité de la période traitée par WACK! puisque jusqu'à la fin des années 1970, ces questions étaient celles qui restaient parmi les plus périphériques au sein du mouvement féministe blanc dominant.

Alors que les années 1990 sont celles précisément où les questions féministes rejoignent les positions culturelles postcoloniales, on constate que c'est une exposition comme Global Feminisms, qui, en enjambant les années 1980, tente d'articuler plus amplement cette cartographie mondiale de l'art et du féminisme contemporain. En proposant la découverte de l'actuelle génération d'artistes femmes, l'exposition souligne les nouveaux impératifs d'une analyse où différences raciales et sexuelles sont indissociées.

\section{Global Feminisms, New Directions in Contemporary Arts : le monde entier est féministe}

15 Que Linda Nochlin, éminente historienne de l'art féministe, à l'origine, avec son célèbre essai «Why Have There Been No Great Women Artists? » $(1970)^{9}$, d'une révolution au sein de la discipline, soit à l'initiative de Global Feminisms, trente ans après sa fameuse exposition Women Artists 1550-1950 organisée avec Ann Sutherland Harris (County Museum of Art de Los Angeles, 1976-1977), pourrait surprendre si l'on ne connaissait d'elle que les thèmes de ses séminaires à la New York University, plus axés aujourd'hui sur la période du XIX ${ }^{e}$ siècle que contemporaine. C'est invitée par Maura Reilly, l'une de ses anciennes étudiantes, conservateur au Elizabeth A. Sackler Center for Feminist Art, nouvellement créé au sein du Brooklyn Museum, que Linda Nochlin s'est associée à cet ambitieux projet. Quatre-vingt cinq artistes originaires des six continents, toutes nées dans les années 1960 ou 1970, ont été sélectionnées mais, en regardant de près les œuvres choisies, utilisant des médiums de représentations aussi divers que la céramique, la broderie, la vidéo, la photographie, la peinture, la sculpture, le dessin ou les nouvelles technologies, un problème de cohérence se pose. S'il est vrai qu'il se retrouve dans toute exposition de groupe, il est d'autant plus aigu dans un projet qui tend à souligner les notions de différences culturelles et à rendre compte de pratiques produites dans 51 pays différents. 
16 La difficulté première réside sans doute dans la façon dont le concept même d'une exposition doit être articulé. Dans leur préface, Maura Reilly et Linda Nochlin exposent ainsi leur intention et leur méthode d'investigation: "Nous n'attendions pas des femmes boliviennes ou pakistanaises d'exposer les spécificités ethniques de leur culture dans leur art, pas plus que nous ne l'aurions attendu d'une artiste américaine. Si cela avait été le cas, nous aurions été dans une pensée à la fois naïve et hégémonique. Toutefois, nous étions très intéressées par l'innovante diversité avec laquelle les femmes de différentes parties du monde déploient consciemment, à partir de leur héritage culturel, une œuvre nouvelle, souvent marquée par une expression visuelle critique $»^{10}$. Alors qu'elles confirment leur ignorance des pratiques produites hors du monde américain ou européen et qu'elles ne souhaitent pas revêtir le rôle de " commissaire-explorateur» (curator as explorator), elles affirment que leurs choix ont été effectués grâce à une recherche intensive, de nombreux voyages mais surtout grâce au dialogue avec des spécialistes de ces aires culturelles méconnues d'elles. Reprenant l'expression de Gerardo Mosquera, elles se positionnent comme des «médiateurs de l'échange culturel ». On peut dès lors se demander de quelle façon le modèle occidental du féminisme peut être - ou non - appliqué aux autres pays du globe et, inversement, considérer l'importance de certains féminismes non-occidentaux (par exemple le féminisme indien) par rapport à la pensée eurocentrique.

Ces interrogations sur le statut de l'art féministe dans une perspective globale et postcoloniale sont particulièrement nécessaires dans le contexte des débats critiques actuels et la présence de créations ignorées du public américain ne peut être qu'un enrichissement indéniable. La conception de l'exposition Global Feminisms, comme celle de WACK! s'est faite toutefois selon des thématiques très générales et réductrices - Life Cycles, Identity, Politics, Emotions - qui, d'une certaine façon, renient la spécificité du projet initial. Les œuvres pouvaient être associées indifféremment à l'un ou à l'autre de ces thèmes qui ont fonction de contenants et qui n'éclairent pas la spécificité géographique, pourtant proposée dans le catalogue, de ces pratiques artistiques féminines. L'appréhension à sa juste valeur d'une pensée féministe contemporaine en devient difficile, souffrant d'une approche évasive en raison de notions qui restent illustratives. La notion de "global ", malgré elle reléguée à un second plan, est devenue presque une donnée accessoire alors qu'elle aurait dû être au centre du concept de l'exposition.

18 Néanmoins, ces maladresses conceptuelles ne peuvent permettre de rejeter entièrement l'idée du projet, car celui-ci venait inaugurer avec courage le premier espace entièrement dédié à l'art féministe au sein d'une institution majeure comme le Brooklyn Museum.

\section{Des œuvres enfin mises en avant}

19 C'est dans ce contexte, au sein même du Elizabeth A. Sackler Feminist Art Center, qu'est installée de façon permanente l'œuvre monumentale de Judy Chicago, The Dinner Party, (1974-1979), restée nomade pendant près de trente ans et pourtant référence incontournable de l'art féministe. Hommage aux femmes célèbres dont trente-neuf d'entre elles offrent leur nom aux assiettes en forme de vulves qui sont posées sur trois tables formant un triangle, The Dinner Party a provoqué, depuis sa création, maintes réactions contradictoires dans le monde de l'art. Son esthétique kitsch et la référence 
au sexe féminin comme image et symbole exclusif de la femme l'a en effet souvent maintenue dans une définition essentialiste, largement remise en cause par les artistes et théoriciennes féministes radicales fondant leurs analyses sur la question du sexe et $\mathrm{du}$ genre comme construction sociale et culturelle. Sa visibilité aujourd'hui constitue toutefois toujours un lien avec la mémoire du féminisme des années 1970 et se situe dans la continuité historique déjà évoquée.

L'année 2008 s'ouvrira avec une nouvelle exposition d'importance, organisée cette fois par deux autres pionnières de l'histoire de l'art féministe, Norma Broude et Mary D. Garrard, à l'American University Museum situé dans le Katzen Arts Center de Washington ${ }^{11}$. Intitulée Claiming Space: Some American Feminist Originators, la manifestation est dédiée au travail d'artistes (dont Judy Chicago, Mary Beth Edelson, Joyce Kozloff, Leslie Labowitz, Suzanne Lacy, Yolanda Lopez, Howardena Pindell, Faith Ringgold, Miriam Schapiro, Carolee Schneemann ou Hannah Wilke) considérées à l'avant-garde de l'art féministe aux États-Unis. Sont privilégiées dans l'exposition les œuvres monumentales les plus représentatives de la protestation politique, du travail sur le corps et enfin celles qui ont marqué par leur force décorative et le plaisir visuel qu'elles provoquent. Toutes ces réalisations contraient à l'époque l'absence de visibilité de l'art des femmes dans la sphère publique.

La recherche dans le champ de l'art féministe permet de revenir de façon ininterrompue sur les origines de celui-ci, prolongeant aujourd'hui, plus que jamais, la réflexion générale sur ce moment crucial, où les gender studies et l'histoire de l'art contemporain peuvent s'unir et transmettre avec une heureuse obstination un héritage dont la validité politique continue de nourrir notre présent.

\section{NOTES}

1. Voir notamment pour les publications récentes : Ella Shohat éd., Talking Visions, Multicultural Feminism in a Transnational Age, New York, 1999 ; Fiona Carson, Claire Pajackzkowska éd., Feminist Visual Culture, Londres, 2001 ; Helena Reckitt éd., Art and Feminism, Londres, 2001 (trad. fr. : Art et féminisme, Paris, 2005) ; Hilary Robinson éd., Feminism-Art-Theory, An Anthology 1968-2000, Londres, 2001 ; Amelia Jones éd., The Feminism and the Visual Culture Reader, Londres, 2003 ; Marsha Meskimmon, Women Making Art, History, Subjectivity, Aesthetics, Londres, 2003.

2. Au sujet des gender et queer studies, voir les textes fondateurs : Monique Witting, The Straight Mind and Other Essays, 1992 (trad. fr. La pensée Straight, Paris, 2001); Eve Kosofsky Sedgwick, Epistemology of the Closet, Berkeley, 1990 ; Judith Butler, Gender Trouble, New York/Londres, 1990 (trad. fr. : Trouble dans le genre: pour un féminisme de la subversion, Paris, 2005) et Leo Bersani, Homos, Cambridge (Mass.), 1995 (trad. fr. : Homos: repenser l'identité, Paris, 1998).

3. Le texte de la conférence inaugurale de Lucy Lippard a été revu par l'auteur sous le titre « No Regrets » pour Art in America, juin-juillet 2007, p. 75-79.

4. WACK! Art and the Feminist Revolution, Cornelia Butler, Lisa Gabrielle Mark éd. (cat. expo., Los Angeles, Museum of Contemporary Art, 2007/Washington D C, Washington Museum of Women in the Arts, 2007/New York, PS1 Contemporary Art Center, 2008/Vancouver, Vancouver Art Gallery, 2008-2009), Boston, The MIT Press, 2007; Global Feminisms, New Directions in Contemporary Art, 
Maura Reilly, Linda Nochlin éd., (cat. expo., New York, Brooklyn Museum, 2007), New York, Merrell, 2007.

5. Nous utilisons ici la traduction littérale de l'anglais « of color ». On dit "women of color » quand il est fait référence aux femmes afro-américaines, hispaniques, asiatiques des États-Unis.

6. Voir Audre Lorde, Sister Outsider, Essays and Speeches, Freedom (Ca), 1984.

7. Les noms des artistes donnés à la suite des intitulés le sont de façon non exhaustive.

8. C'est encore une fois le catalogue qui permet de combler les absences de l'exposition : un essai de Catherine Lord, « Their memory is playing tricks on her: Notes toward a Calligraphy of Rage » (p. 441-457), propose une lecture de la confrontation entre le féminisme et l'homosexualité féminine; Richard Meyer dans «Hard Target: Male Bodies, Feminist Art, and the Force of Censorship in the 1970s » (p. 363-383) analyse la relation du féminisme à la sexualité masculine et à la censure subie par certaines artistes qui s'y sont intéressées. Enfin,Valerie Smith adresse un témoignage sur le féminisme afro-américain avec « Abundant Evidence: Black Women Artists of the 1960s and 1970s » (p. 401-413).

9. Linda Nochlin, « Why Have There Been No Great Women Artists? », dans Art News, 1970, 69/9, p. 22-39 et 62-71 ; trad. fr. : « Pourquoi n'y a-t-il pas eu de grandes artistes femmes ? », dans Linda Nochlin, Femmes, art et pouvoir et autres essais, Nîmes, 1993, p. 201-244.

10. «We did not expect women from Bolivia or Pakistan to exhibit specific traits in their art, any more than we expected the same from an artist from the U.S. ; to do so would have been naïve and patronizing. Yet we were open to, and very interested in, the varying and innovative ways that women from diverse parts of the world self-consciously deployed the visual culture they had inherited to create new, often critical visual expressions", Maura Reilly, Linda Nochlin, «Curators' Preface », dans Global Feminisms, 2007, p. 11.

11. Claiming Space: Some American Feminist Originators (6 novembre 2007-27 janvier 2008) se déroule conjointement à l'exposition WACK! à Washington (21 septembre-16 décembre 2007).

\section{INDEX}

Keywords : feminist art, exhibitions, women artists, feminist art history, activism, historiography, gender studies

Mots-clés : art féministe, expositions, femmes artistes, histoire de l'art féministe, activisme, historiographie

Index géographique : États-Unis

Index chronologique : 1900, 2000

\section{AUTEURS}

\section{ELVAN ZABUNYAN}

Université Rennes II, MASCIPO, CNRS 